\title{
EVALUACIÓN DE EVENTOS DEPORTIVOS: EL CASO DEL CAMPEONATO DE ESPAÑA DE DESCENSO DE CAÑONES 2019
}

\section{EVALUATION OF SPORTS EVENTS: THE CASE OF THE SPANISH CANYON DESCENT CHAMPIONSHIP 2019}

Rojo Ramos, J. ${ }^{1 A B C D}$; Cerro Herrero, D. ${ }^{1 A B C D}$; Madruga Vicente, M. ${ }^{1 A B C D}$; Prieto Prieto, J. ${ }^{2 A B C D}$ ${ }^{1}$ Rojo Ramos, J. Facultad de Ciencias del Deporte, Universidad de Extremadura, España, jorgerr@unex.es ${ }^{1}$ Cerro Herrero, D. Facultad de Formación del Profesorado, Universidad de Extremadura, España, davidcerro@unex.es

${ }^{1}$ Madruga Vicente, M. Facultad de Formación del Profesorado, Universidad de Extremadura, España, miguelmadruga@unex.es

2Prieto Prieto, J. Facultad de Educación, Universidad de Salamanca, España, josueprieto@usal.es

Responsabilidades

${ }^{A}$ Diseño de la investigación. ${ }^{B}$ Tratamiento de datos. ${ }^{C}$ Redactor del trabajo. ${ }^{D}$ Coordinador de la investigación.

\section{RESUMEN}

El crecimiento que han experimentado en los últimos años los eventos deportivos exige a los organizadores ser sensibles a las opiniones de los participantes, con el propósito de elaborar estrategias adecuadas para una gestión exitosa de los eventos deportivos. El objetivo del estudio consistió en evaluar la calidad e impacto percibido por los deportistas del Campeonato de España de Descenso de Cañones de 2019. Participaron 52 deportistas, $74 \%$ hombres y $26 \%$ mujeres, con una edad media de $36.26 \pm 8.86$ años. Se utilizó un cuestionario para la recolección de datos con tres secciones: datos socio-deportivos, calidad-satisfacción, e impacto-repercusión. Los participantes reportaron una alta satisfacción con el evento, y perciben el acontecimiento como generador de impactos positivos en el territorio en que se celebra. Con relación al impacto económico, la estimación de gasto generado por los participantes en el evento y reportada directamente por los encuestados fue de 252,61€. La evaluación de la calidad e impacto, es una estrategia útil para los organizadores de eventos deportivos, tanto en la mejora y diseño de sus 
servicios, así como muestra de evidencia de las inversiones realizadas por patrocinadores y administraciones.

\section{PALABRAS CLAVE}

eventos deportivos, calidad percibida, impacto, gasto, descenso de cañones.

\section{ABSTRACT}

The growth experienced by sports events during last years demands that managers need to be sensitive to the opinions of participants in order to develop appropriate strategies for the successful management of those sports events. This study aimed to evaluate the quality and perceived impact by the athletes of the Canyoning Championship of Spain in 2019. Fifty-two athletes participated, $74 \%$ men and $26 \%$ women, with a mean age of $36.26 \pm 8.86$ years. A questionnaire including three sections was used for data collection: socio-sports data, qualitysatisfaction, and impact-repercussion. Participants reported a high level of satisfaction with the event, and perceived the event as a generator of positive impacts on the territory where it was held. In relation to the economic impact, the estimated expenditure generated by participants on the event and reported directly by the respondents was $€ 252.61$. The evaluation of quality and impact is a useful strategy for the organisers of sports events, both in the improvement and design of their services and as well as evidence of the investments made by sponsors and administrations.

\section{KEY WORDS}

sports events, perceived quality, impact, expenditure, canyon descent.

\section{INTRODUCCIÓN}

El sector deportivo ha experimentado una evolución muy importante en los últimos años a nivel nacional, desde el tipo de actividades practicadas hasta factores que tienen influencia sobre la economía (1). En particular, la participación en eventos deportivos se ha convertido en una de las actividades de ocio activo más populares de la población en los últimos años $(2,3)$. En España se ha producido un crecimiento notable del número de eventos deportivos principalmente en el ámbito no profesional que se celebran tanto a nivel local, como autonómico, 
nacional e internacional (4). Esta globalización y diversificación de los eventos deportivos queda reflejada en un crecimiento de la literatura científica relacionada con la temática, que evalúan no solo la calidad de estos acontecimientos como aspecto clave para la mejora de los servicios ofrecidos por los organizadores (5), sino también el impacto económico, social o turístico que se produce en los territorios donde se desarrollan (6), dado el potencial de este recurso para generar importantes beneficios (7).

Evaluar la calidad de los eventos exige a los organizadores ser sensibles a las opiniones de los participantes, con el propósito de conocer ciertos elementos percibidos por los usuarios, y elaborar estrategias adecuadas para una gestión exitosa de los eventos deportivos a partir de los resultados (8). En general, la calidad percibida del servicio, como lo puede ser un evento deportivo, puede definirse como "la diferencia entre lo que se espera del servicio y lo que un consumidor percibe o recibe del mismo" (9). Este concepto se relaciona estrechamente con otros constructos como el valor percibido, la satisfacción o las intenciones futuras $(10,11)$. Estos factores suscitan el interés de los organizadores para mejorar su gestión del evento.

El análisis de los niveles de satisfacción y calidad percibida en los eventos deportivos ha estado focalizado fundamentalmente en la práctica deportiva profesional, y han sido evaluados principalmente desde la perspectiva de los asistentes o espectadores (12). Cada vez son más los estudios en la literatura que realizan análisis de la calidad percibida y otras variables como la satisfacción o el valor percibido en eventos de diferentes modalidades deportivas (13-15). Sin embargo, la diversidad de métodos y herramientas utilizadas para evaluar la calidad del evento, manifiestan amplia variabilidad de resultados. A pesar de ello, estudios previos señalan que estas variables deben ser analizadas, con el objetivo de añadir criterios de eficiencia, para mejorar la gestión de los eventos deportivos (8), favoreciendo que los organizadores conozcan las necesidades de sus clientes para mejorar la calidad del servicio (5). Entre las dimensiones que deben ser evaluadas en los eventos deportivos en relación a la calidad percibida, destacan algunas como son: a) interacción personal, comunicación, servicios complementarios e infraestructura logística (16), b) servicio global, satisfacción general, valor percibido e intenciones futuras (17), o c) aspectos tangibles, personal, accesibilidad y servicios complementarios (13).

Por otra parte, la trascendencia económica que han alcanzado los eventos requiere también de la evaluación del impacto socioeconómico que produce en la zona (18). En este sentido, el impacto que generan los eventos deportivos puede ser tanto positivo como negativo, sobre todo en el caso de aquellos de menor envergadura, ya que, aunque no 
generan tanto interés social o mediático, sí pueden llegar a producir beneficios (19). Los estudios realizados hasta ahora se han centrado, en la mayoría de los casos, en la visión de los espectadores (20-22) y sobre todo en la percepción de los residentes de la localidad donde se organiza el evento (23-25), y los estudios centrados en la percepción de los deportistas participantes en el evento son escasos.

La evaluación del impacto socioeconómico de los eventos deportivos se inició con el estudio de macro-eventos como los Juegos Olímpicos, Campeonatos del Mundo y Universiadas (26-28). Poco a poco este interés se ha extendido a eventos de menor magnitud, sin embargo, existe un número reducido de estudios sobre eventos de tamaño medio o pequeños eventos, y apenas existen estudios de impacto en aquellos eventos que se desarrollan en el medio rural. Aún así, recientemente diversos estudios en España han evaluado el impacto económico de algunos eventos deportivos: Universiada de Invierno de Granada (13), Campeonato de España Máster Natación en Pontevedra (19), Gran Premio de Motociclismo de Jerez de la Frontera (29), Open 500 de Tenis de Valencia (30) y Trail Walker Intermon-Oxfam (31). De manera particular, en la comunidad autónoma de Extremadura también se han analizado algunos eventos deportivos, como el Campeonato de España de Ciclismo en Cáceres el año 2015 y Descenso del Alagón del año 2013 (7), World Padel Tour en Cáceres $(32,33)$, Vuelta al Baluarte (34), o eventos de pequeña escala en la Comarca del Valle de Jerte (35).

Es bien sabido, que los eventos incentivan el desarrollo socioeconómico local, favorecen la creación de empleo, promueven la creación y mejora de infraestructuras, y todo ello beneficia al turismo y a la población local (29). Entre estos beneficios, cabe destacar la capacidad de atraer flujos turísticos a un destino, concentrados en un periodo de tiempo concreto. Tal y como señalan Henderson et. al., esa capacidad de atraer un gran número de turistas a la zona del evento, suele ir asociada a un gasto económico por parte del turista (36). Sánchez y col. (37) enumeran los factores que influyen en la generación de impacto económico, entre los que destacan, el tipo de participación (individual o equipo), el tipo de competición, el ámbito geográfico, la duración del evento, los participantes, la federación, los espectadores, el espacio, las infraestructuras, la periodicidad de celebración y la tipología del evento.

En relación al tipo de evento deportivo, parece evidente que los de pequeña y mediana magnitud no deberían generar la misma repercusión económica que los de gran escala (30). Sin embargo, Veltri, Miller y Harris (38) matizan que este tipo de eventos pueden generar proporcionalmente más beneficios económicos si se celebran en localidades pequeñas o medianas que si tienen lugar en grandes 
ciudades. En este sentido, en España son cada vez más numerosos los eventos deportivos de menor magnitud, y una importante cifra de estos se desarrollan en localidades pequeñas y medianas, y corresponden a modalidades deportivas desarrolladas en el medio natural, tales como: carreras por montaña, orientación, escalada, descenso de barrancos 0 cañones, mountain bike, senderismo, entre otras (39).

Por todo ello, convenimos de la importancia de evaluar los eventos deportivos, tanto desde la perspectiva de calidad como herramienta de los organizadores para la mejora de los servicios, como del impacto para valorar la repercusión de estos y el beneficio en los territorios. En este sentido, se plantea el presente estudio, que tiene por objetivo analizar la calidad percibida y el impacto repercusión de un evento deportivo de pequeña magnitud desde el punto de vista de los participantes. Para ello se realizó un estudio de caso en el Campeonato de España de Descenso de Cañones de 2019.

\section{MATERIAL Y MÉTODOS}

El diseño de la investigación responde a un modelo descriptivo cuantitativo mediante el uso de la encuesta como técnica para la obtención de los datos y su posterior análisis. A continuación se describen los diferentes apartados del diseño.

\section{Participantes}

La población objeto de estudio estaba constituida por los participantes del Campeonato de España de Descenso de Cañones de la Federación Española de Montaña y Escalada de 2019, celebrado en la localidad de Navaconcejo, en la comarca del Valle del Jerte (Extremadura). Un total de 52 personas participaron en dicho campeonato, de las cuales $46(88,46 \%)$ accedieron a participar en el presente estudio. La edad media de los participantes fue de $36,26+8,86$ años. Del total de encuestados, $41(91,1 \%)$ eran no residentes en la comarca.

Con relación al per il de los encuestados en las variables relacionadas con la estancia, un $95,7 \%$ de los declara que el evento es el motivo de la estancia en la comarca, y un $91,3 \%$ de los participantes no residen en ella. Además, el $87 \%$ de los participantes acuden acompañados de 1-2 personas $(M=1,76, d t=1,405)$, de las cuales al menos una $(M=1,26$, $\mathrm{dt}=1,373)$ no era participante también del evento. Un total de 41 participantes pernoctaron en la localidad o comarca con una media por persona de 2.46 noches $(\mathrm{dt}=1.373)$, y la suma de 113 pernoctas en el total de encuestados. El alojamiento mayoritario elegido por los participantes fueron camping (43.5\%) y casa rural $(32.6 \%)$. 


\section{Tabla 1. Características de los participantes}

\begin{tabular}{|c|c|c|c|}
\hline \multicolumn{2}{|l|}{ Variables } & Frecuencia & Porcentaje \\
\hline \multirow{2}{*}{ Sexo } & Hombre & 34 & 73,9 \\
\hline & Mujer & 12 & 26,1 \\
\hline \multirow{3}{*}{ Edad (grupos) } & Entre 18 y 34 años & 20 & 43,5 \\
\hline & Entre 35 y 44 años & 16 & 34,8 \\
\hline & De 45 en adelante & 10 & 21,7 \\
\hline \multirow{2}{*}{ Nivel de estudios } & No universitarios & 22 & 47,8 \\
\hline & Universitarios & 24 & 52,2 \\
\hline \multirow{4}{*}{ Procedencia (provincia) } & Málaga & 15 & 32,6 \\
\hline & Granada & 8 & 17,4 \\
\hline & Cáceres & 8 & 17,4 \\
\hline & Otras & 15 & 32,6 \\
\hline \multirow{5}{*}{ Situación laboral } & Activo/a & 35 & 76,1 \\
\hline & Amo/a de casa & 1 & 2,2 \\
\hline & Desempleado & 3 & 6,5 \\
\hline & Estudiante & 6 & 13,0 \\
\hline & Jubilado/Pensionista & 1 & 2,2 \\
\hline \multirow{2}{*}{ Federado (cañones) } & $\mathrm{Si}$ & 41 & 89,1 \\
\hline & No & 5 & 10,9 \\
\hline \multirow{4}{*}{$\begin{array}{l}\text { Entrenamiento (horas de prác- } \\
\text { tica semanal) }\end{array}$} & Entre 0 y 3 horas & 11 & 23,9 \\
\hline & Entre 3 y 6 horas & 16 & 34,8 \\
\hline & Entre 6 y 10 horas & 13 & 28,3 \\
\hline & Más de 10 horas & 6 & 13,0 \\
\hline \multirow{2}{*}{ Modalidad participación } & Individual & 27 & 58,7 \\
\hline & Equipos & 19 & 41,3 \\
\hline \multirow{2}{*}{ Evento es motivo de estancia } & $\mathrm{Si}$ & 44 & 95,7 \\
\hline & No & 2 & 4,3 \\
\hline \multirow{2}{*}{ Residente en la comarca } & $\mathrm{Si}$ & 4 & 8,7 \\
\hline & No & 42 & 91,3 \\
\hline \multirow{2}{*}{ Acude acompañado } & $\mathrm{Si}$ & 40 & 87,0 \\
\hline & No & 6 & 13,0 \\
\hline
\end{tabular}




\begin{tabular}{|c|c|c|c|}
\hline Variables & & Frecuencia & Porcentaje \\
\hline \multirow{4}{*}{ Número de acompañantes } & Ninguno & 6 & 13,0 \\
\hline & Uno & 20 & 43,5 \\
\hline & Dos & 10 & 21,7 \\
\hline & Tres o más & 10 & 21,7 \\
\hline \multirow{4}{*}{ Acompañantes no participantes } & Ninguno & 16 & 34,8 \\
\hline & Uno & 15 & 32,6 \\
\hline & Dos & 8 & 17,4 \\
\hline & Tres o más & 7 & 15,2 \\
\hline \multirow{2}{*}{ Pernocta en la comarca } & Si & 41 & 89,1 \\
\hline & No & 5 & 10,9 \\
\hline \multirow{4}{*}{ Número de noches de pernocta } & Ninguno & 5 & 10,9 \\
\hline & Uno & 2 & 4,3 \\
\hline & Dos & 17 & 37,4 \\
\hline & Tres o más & 22 & 47,9 \\
\hline \multirow{6}{*}{ Tipo de alojamiento } & Camping & 20 & 43,5 \\
\hline & Casa Rural & 15 & 32,6 \\
\hline & Hostal & 2 & 4,3 \\
\hline & Hotel & 4 & 8,7 \\
\hline & Furgoneta & 2 & 4,3 \\
\hline & Vivienda propia & 3 & 6,5 \\
\hline
\end{tabular}

\section{Instrumento}

Para la recogida de datos se diseñó un instrumento específico para el estudio, adaptado de los instrumentos empleados en los estudios de Angosto (8) y Jiménez (33). Los dos instrumentos utilizados como base mostraron en sus estudios de validación una fiabilidad de la escala que puede considerarse satisfactoria. Por una parte, CAPPEP V2.0 (8) contó con las siguientes fiabilidades factor 1 (servicios complementarios) $\alpha=0,867$, factor 2 (comunicación) $a=0,726$, factor 3 (infraestructura logística) $a=0,733$ y factor 4 (interacción personal) $a=0,804$. La fiabilidad total del cuestionario obtuvo un valor excelente de 0,915. El porcentaje total de varianza explicada fue igual al $68.82 \%$. Por otro lado los instrumentos desarrollados por Jiménez (33) destaca que son estudios en etapas iniciales de desarrollo de escalas, los cuales han sido sometidos a diversas pruebas para analizar la validad y fiabilidad de las variables, pero aun es complicado que sean aplicables a modalidades deportivas diversas. 
El cuestionario empleado quedó integrado por 50 ítems divididos en tres secciones. La primera sección recoge los datos del perfil socio-deportivo del participante, con 8 ítems (sexo, edad, procedencia, nivel de estudios, situación laboral, condición de federado, práctica semanal, modalidad de participación). La segunda sección, formada por 22 ítems, organizado en dos escalas, que valora aspectos referidos a la calidad percibida y satisfacción del evento (CS) con 13 ítems, y el impacto y repercusión del evento (IR) con 9 ítems. Se empleó una escala tipo Likert con 5 puntos de anclaje (1=máximo desacuerdo, 5=máximo acuerdo). La tercera sección formada por 20 ítems, que están relacionados con la estimación de gasto del participante en el evento, tales como: motivo de estancia, número de pernoctas, tipo de alojamiento, consumos, y gastos totales.

\section{Procedimiento}

Previamente a la recogida de datos, se contactó con el organizador del evento deportivo, con el objetivo de informar del propósito del estudio y la forma de participación, así como para obtener el consentimiento para participar en el estudio y autorizar encuestar a los deportistas. La recogida de datos se realizó aplicando el cuestionario diseñado a través del método de encuesta personal. Esta encuesta fue realizada por un encuestador formado con relación a la metodología utilizada, a sus objetivos y a la correcta aplicación del cuestionario. Todos los datos ueron tratados con la finalidad de eliminar las encuestas no válidas y en las cuales se encontraron valores erróneos. El cuestionario fue elaborado y administrado en soporte digital, utilizando la herramienta Google Forms, seleccionada en base a criterios de funcionalidad y operatividad para los participantes, y a su practicidad, pues permite guardar las respuestas directamente en una hoja de cálculo y así facilitar la transformación de estas para realizar un análisis estadístico adecuado. La recogida de datos tuvo lugar el último día de celebración del evento. Los participantes en el estudio recibieron información detallada de la finalidad de este estudio durante el evento. Asimismo, ueron asistidos en grupos reducidos por un técnico experto de evaluación sobre las dudas existentes y en la comprensión de los ítems. Completaron el cuestionario de forma anónima, facilitado mediante enlace url y con la presencia del encuestador para resolver dudas. El tiempo destinado para responder al cuestionario fue de 10-15 minutos.

\section{Análisis estadístico}

En primer lugar, se realizó un análisis descriptivo de las variables sociodemográficas $\mathrm{d}$ e $\mathrm{l}$ os $\mathrm{p}$ articipantes $\mathrm{d}$ el estudio $\mathrm{m}$ ediante la 
frecuencia y porcentajes. Seguidamente, para probar la asociación entre las variables estudiadas se optó por un análisis de correlaciones bivariadas mediante coeficiente de Pearson y nivel de significación al nivel $<0,05$ y $<0,01$, entre las variables dependientes y la totalidad de ítems del instrumento. Para la determinación de diferencias entre las variables de estudio, se realizó un análisis mediante la Prueba T para la variables dicotómicas y análisis de varianza de un factor (ANOVA) para las variables politómicas. La significación estadística ue $p<0.05$. Todos los análisis fueron realizados utilizando el paquete estadístico SPSS versión 23.0 para Windows (IBM, Armonk, Nueva York).

\section{RESULTADOS}

\section{Calidad percibida y satisfacción con el evento}

La tabla 2 muestra las puntuaciones medias de todos los ítems del instrumento de la escala de CS. Los ítems más valorados fueron la intención futura de participar $(4,9 \pm 0,3)$, la atención con amabilidad de los voluntarios $(4,9 \pm 0,4)$. En cambio, los ítems con menor valoración fueron la promoción, difusión e información del evento $(3,1 \pm 0,3)$, los puntos de avituallamiento $(3,3 \pm 1,5)$ y los servicios de apoyo $(3,3 \pm 1,4)$. El ítem de valoración general del evento reportó una puntuación media de $4,3+0,5$. Fue generado un factor de calidad-satisfacción que aglutinó todas ítems de la subescala con una puntuación media de 4,4+0,4.

\section{Tabla 2: Puntuaciones de los ítems de calidad-satisfacción con el evento.}

\begin{tabular}{lcc}
\hline Ítems & Media & DT \\
\hline Escala de calidad-satisfacción con el evento deportivo & & 1,25 \\
$\begin{array}{l}\text { CS1. La carrera cuenta con una buena promoción y difusión, aportando suficiente } \\
\text { información práctica sobre la misma. }\end{array}$ & 3,07 &, 69 \\
CS2. He recogido fácilmente el dorsal sin realizar mucha espera. & $4,72,83$ \\
CS3. El personal de seguridad y tráfico está dispuesto a ayudar/dar consejos. & $4,59,34$ \\
CS4. El personal de la organización está dispuesto a ayudar/dar consejos. & 4,87 &, 38 \\
CS5. Los voluntarios atienden con amabilidad. & 4,89 &, 44 \\
CS6. El recorrido del descenso está bien equipado y es seguro & 4,83 & 1,53 \\
CS7. El campeonato cuenta con puntos de avituallamiento, y son adecuados & 3,30 \\
CS8. El campeonato cuenta con servicios de apoyo suficientes (baños, vestuarios, & 3,33 & 1,45 \\
guardarropa, stands, etc.). & 4,30 &, 84 \\
CS9. En general, el servicio ofrecido por la organización es adecuado. &, 57 \\
CS10. En general, me parece que la carrera tiene una buena relación calidad-precio. & 4,65 & ,
\end{tabular}




\begin{tabular}{lll} 
Ítems & Media & DT \\
\hline CS11. Estoy dispuesto a continuar volviendo al campeonato en próximas ediciones. & 4,89 &, 32 \\
CS12. Recomendaré la asistencia a la carrera a mis amigos y parientes. & 4,85 &, 42 \\
CS13. ¿Qué puntuación le daría al evento en su conjunto? & 4,28 &, 50 \\
CS $_{\text {total }}$ Puntuación media de los ítems de calidad-satisfacción & 4,35 &, 43
\end{tabular}

El análisis de correlaciones bivariadas entre las variables sociodemográficas y los ítems de la escala de CS (tabla 3), mostró asociaciones bajas y moderadas en diversos ítems. En relación al sexo, mostró una asociación baja $(r=.319, p<.01)$ en la percepción general de la adecuación del servicio ofrecido (CS09), con puntuación más elevada en el grupo de mujeres. En relación a la variable edad (años del participante), se observa una relación positiva $(p<.05)$ con las puntuaciones otorgadas en el ítem referido a la relación calidad-precio (CS10), de modo que a mayor edad mejor valoración en todos ellos. En cambio, en el análisis de los grupos de edad establecidos, las diferencias fueron observadas en el ítem CS1 $(p<.01)$ entre el grupo de $45+y$ los otros dos grupos (pero no entre ellos). Por último, en relación a las variables deportivas, no se encontraron asociaciones con el nivel entrenamiento, y tan solo en la recogida del dorsal (CS2) con la modalidad de participación $(\mathrm{p}<.05)$.

Tabla 3. Correlación entre variables sociodemográficas y los ítems de calidad percibida y satisfacción del evento.

\begin{tabular}{llllllll} 
Ítems & 1 & 2 & 3 & 4 & 5 & 6 & 7 \\
\hline CS1 &,- 128 &, $398^{* *}$ &, 191 &, 031 &,- 075 &, 145 &,- 009 \\
CS2 &,- 101 &, 130 &,- 014 &, 032 &,- 163 &,- 165 &,$- 365^{*}$ \\
CS3 &,- 178 &, 233 &,- 057 &,- 067 &, 006 &,- 168 &,- 008 \\
CS4 &,- 230 &,- 025 &, 275 &,- 121 &,- 135 &, 055 &, 194 \\
CS5 &,- 040 &, 217 &, 071 &,$- 433^{* *}$ & 0,101 &,- 028 &, 126 \\
CS6 &, 104 &,- 112 &, 118 &, 087 &,- 140 &,- 029 &, 235 \\
CS7 &,- 109 &,- 167 &, 106 &, 220 &,- 162 &,- 121 &,- 168 \\
CS8 &,- 245 &, 259 &, 249 &, 109 &,- 080 &,$- 305^{*}$ &,- 006 \\
CS9 &,$- 319^{*}$ &, 102 &, 141 &,- 018 &,- 044 &,- 195 &, 012 \\
CS10 &,- 104 &, $325^{*}$ &,- 051 &,$- 455^{* *}$ & 0,092 &,- 085 &, 206 \\
CS11 &, 111 &,- 008 &,- 195 &,- 227 &,- 102 &, 109 &, 009 \\
CS12 &, 259 &,- 035 &,- 141 &,- 247 &,- 040 &, 275 &,- 118 \\
CS13 &,- 061 &, 047 &, 019 &,- 081 &, 083 &, 002 &,- 033
\end{tabular}

1 Sexo; 2 Grupos Edad; 3 Estudios; 4 Laboral; 5 Federado; 6 Entreno; 7 Modalidad.

${ }^{*} p<.05,{ }^{* *} p<.01$. 


\section{Impacto y repercusión percibida del evento}

En relación a los ítems de la subescala de IR (tabla 4), las puntuaciones más elevadas fueron obtenidas en la percepción de no generar problemas de tráfico y seguridad $(4,4 \pm 0,8)$, de incremento del turismo antes y después del evento $(4,3 \pm 1,0)$ y no supone pérdidas económicas $(4,3 \pm 0,7)$. Los ítems menos valorados fueron las ventajas económicas concentradas en pocas empresas o personas $(3,0 \pm 1,0)$ y el aumento de empleo $(3,3 \pm 1,1)$. La puntuación media en el factor de impacto generado

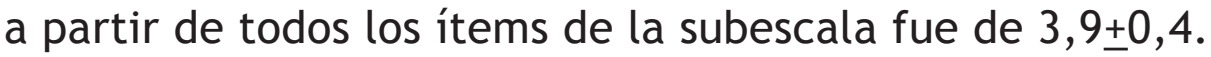

Tabla 4: Puntuaciones de los ítems de impacto-repercusión en la localidad.

\begin{tabular}{|c|c|c|}
\hline Ítems & Media & DT \\
\hline \multicolumn{3}{|l|}{ Escala de impacto-repercusión del evento en la localidad } \\
\hline IR1. Aumenta en reconocimiento y la promoción & 4,13 & 1,24 \\
\hline IR2. Representa un elemento de orgullo y satisfacción & 4,07 & 1,20 \\
\hline IR3. Genera problemas de tráfico y seguridad $\beth$ & 4,41 & 81 \\
\hline IR4. Altera el ritmo habitual y perjudica otras actividades $I$ & 4,07 & ,88 \\
\hline IR5. Provoca un incremento del turismo durante y después del evento & 4,26 & ,96 \\
\hline IR6. Aumenta el empleo & 3,28 & 1,07 \\
\hline IR7. Supone pérdidas económicas, debido a inversión mayor que beneficios $\neg$ & 4,26 & ,74 \\
\hline $\begin{array}{l}\text { IR8. Las ventajas económicas se concentran en unas pocas empresas y per- } \\
\text { sonas } 7\end{array}$ & 2,98 & ,95 \\
\hline IR9. El gasto público es necesario para la celebración de este tipo de eventos & 3,59 & 1,36 \\
\hline $\mathrm{IRt}_{\text {otal }}$ Puntuación media de los ítems de impacto-repercusión & 3,89 & 0,44 \\
\hline
\end{tabular}

El análisis de correlaciones bivariadas entre las variables sociodemográficas y los ítems de la escala de IR (tabla 5), mostró asociaciones bajas y moderadas en diversos ítems. En relación al sexo, mostró una asociación moderada $(r=.411, p<.01)$ con la percepción de alteración del ritmo habitual y de actividades (IR4), otorgando a este aspecto una puntuación más negativa por parte de los hombres. En relación a la variable edad (años del participante), se observa una relación positiva $(p<.05)$ con las puntuaciones otorgadas en los ítems referidos la concentración de los beneficios en pocos (IR08) y la necesidad de gasto público (IR09), de modo que a mayor edad mejor valoración en todos ellos. En cambio, en análisis de los grupos de edad establecidos, las diferencias fueron observadas en el ítem IR8 $(p<.05)$ entre el grupo de $45+y$ los otros dos grupos (pero no entre ellos). 
En relación a la condición de federado, se observa una asociación baja $(r<.04, p<.05)$ con los ítems de la escala de impacto-repercusión referidos a la alteración del ritmo habitual (IR4) más valorado por los participantes no federados, y también respecto al ítem de aumento del empleo (IR6), siendo los deportistas no federados que más valoran este aspecto. Por último, en relación a la variable de entrenamiento, se encontraron diferencias en IR3 $(\mathrm{p}<.05)$ entre los grupos de 6-10 horas y $<10$ horas y IR7 $(p<.05)$ entre los grupos de 0 -3horas y 3 -6horas. En relación a la modalidad de participación en IR7 $(\mathrm{p}<.05)$.

Tabla 5. Correlación entre variables sociodemográficas y los ítems de impacto y repercusión del evento.

\begin{tabular}{llllllll} 
Ítems & 1 & 2 & 3 & 4 & 5 & 6 & 7 \\
\hline IR1 &,- 018 &,- 039 &, 173 &, 063 &, 077 &, 040 &, 127 \\
IR2 &,- 009 &,- 008 &, 199 &,- 029 &, 098 &, 002 &, 140 \\
IR3 &,- 065 &,- 101 &, 114 &, 070 &,- 181 &,- 190 &, 064 \\
IR4 &,$- 411^{* *}$ &, $\mathbf{3 1 0 *}$ &,- 028 &, 066 &,$- 347^{*}$ &,- 228 &,- 164 \\
IR5 &, 269 &,- 071 &, 080 &,- 050 &, 200 &, 126 &, 236 \\
IR6 &, 018 &,- 058 &, 133 &,- 090 &, $303^{*}$ &, 128 &, 110 \\
IR7 &,- 126 &, 023 &,- 075 &, 185 &, 066 &,- 111 &,$- 298^{*}$ \\
IR8 &,- 014 &, $408 * *$ &,- 114 &, 226 &, 230 &,- 064 &,- 074 \\
IR9 &,- 109 &, $330 *$ &, 159 &,- 096 &, 055 &,- 004 &,- 104 \\
IR &,- 107 &, 216 &, 198 &, 059 &, 156 &,- 049 &, 036 \\
\hline total &,- 056 &
\end{tabular}

1 Sexo; 2 Grupos Edad; 3 Estudios; 4 Laboral; 5 Federado; 6 Entreno; 7 Modalidad.

${ }^{*} \mathrm{p}<.05,{ }^{* *} \mathrm{p}<.01$.

\section{Estimación de gasto de los participantes}

La tabla 6 contempla el gasto medio estimado de los participantes en los diversos conceptos desde tres escenarios diferentes, bajo, medio y alto. Los encuestados fueron preguntados por el gasto en un rango (0; $<30 ;<60 ;<90 ;<120 ;<150 ;>150$ ). En el escenario bajo, las respuestas obtenidas fueron multiplicadas por el valor mínimo del rango $(0,10$, $40,70,100,130,160)$. En el escenario medio, las respuestas fueron multiplicadas por el valor medio del rango $(0,20,50,80,110,140,170)$. Finalmente, en el escenario alto, las respuestas fueron multiplicadas por el valor máximo del rango $(0,30,60,90,120,150,180)$.

De forma general, el gasto medio estimado por participante, oscila en un rango de 199,48 a 295,57 euros, siendo los gastos mayoritarios los 
correspondientes a las categorías de alojamiento (de 56,74 a 73,70 ), combustible (de 50,00 a 69,13), comida y cena (de 37,61 a 56,74 ) y bares (de 31,30 a 50,87). Los gastos de menor importe son acometidos en ropa (de 3,26 a 4,57) y museos/visitas (de 3,91 a 7,83).

Tabla 6. Estimación de gastos por participante en tres escenarios (en $€$ ).

\begin{tabular}{lcccccc} 
& \multicolumn{2}{c}{ Escenario 1 } & \multicolumn{2}{c}{ Escenario 2 } & \multicolumn{2}{c}{ Escenario 3 } \\
\cline { 2 - 7 } & Media & DT & Media & DT & Media & DT \\
\hline Alojamiento & 56,74 & 40,28 & 65,22 & 42,57 & 73,70 & 45,04 \\
Combustible & 50,00 & 37,54 & 59,57 & 38,18 & 69,13 & 38,92 \\
Comidas / cenas & 37,61 & 32,94 & 47,17 & 33,51 & 56,74 & 34,19 \\
Bares & 31,30 & 28,25 & 41,09 & 28,54 & 50,87 & 28,89 \\
Souvenirs & 5,65 & 7,20 & 10,65 & 11,62 & 15,65 & 16,42 \\
Desplazamientos (taxi, autobús) & 10,22 & 30,95 & 12,61 & 33,69 & 15,00 & 36,74 \\
Museos / visitas & 3,91 & 14,98 & 5,87 & 17,46 & 7,83 & 20,43 \\
Otros & 0,78 & 2,54 & 1,43 & 5,00 & 2,09 & 7,48 \\
Ropa & 3,26 & 19,21 & 3,91 & 20,92 & 4,57 & 22,78 \\
Prensa & 0,00 & 0,00 & 0,00 & 0,00 & 0,00 & 0,00 \\
Total por participante & 199,48 & 114,59 & 247,52 & 119,59 & 295,57 & 125,36
\end{tabular}

La estimación de gasto en el evento reportada directamente por los encuestados fue de $252,6+193,6$ euros, un valor próximo al calculado en el escenario medio ( $d i f M=-5,09, d t=178,20$ ), no hallando diferencias significativas entre ambas puntuaciones $(p=.636)$. El gasto medio de los participantes en el evento difiere en la comparación de residentes $(80,0+24,5)$ y no residentes $(263,5+112,5)$, siendo superior en măs de un $300 \%$ en los primeros, y determinado principalmente por el gasto de alojamiento y combustible. Además, los participantes estimaron un gasto medio en este evento de 204,1 euros $(\mathrm{dt}=106,385)$.

Teniendo en cuenta el promedio total de gastos de los encuestados, el gasto total calculado para los 52 participantes ( 5 residentes y 47 no residentes) fue de $12.783,6$ euros. El gasto en inscripciones, informado por el organizador, fue de 1084,0 euros, con un total de 30 individuales $(20,0 €)$ y 22 equipos $(22,0 €)$.

\section{DISCUSIÓN}

Los objetivos de este estudio de caso fueron analizar la calidadsatisfacción y el impacto-repercusión percibido por los deportistas 
participantes del Campeonato de España de Descenso de Cañones de 2019.

Pese a que el objetivo del estudio no pretende definir las características del perfil de competidores en descenso de Cañones, el evento analizado permite definir este perfil predominado por hombres, con una edad media de 36 años, con nivel de estudios medio-alto, y situación laboral activa. La procedencia de los participantes del evento fue principalmente de Andalucía, por lo que los datos referidos deben ser tomados con cautela. En cuanto a las características deportivas, predomina la condición de federado, y su nivel de entrenamiento es de 3 a 10 horas semanales.

Respecto a la satisfacción de los participantes en el Campeonato de España, ha sido elevada con una media de 4,31 sobre un valor máximo de 5 , detectando como aspectos mejorables en los servicios ofrecidos a los participantes, tales como avituallamiento, vestuarios, duchas, guardarropa o stands. Los resultados obtenidos son coherentes con estudios previos $(15,40-42)$, donde los participantes de los eventos deportivos acostumbran a reportar un nivel de satisfacción elevado. No obstante, es preciso tener en cuenta que la comparación entre modalidades tiene muchas limitaciones, ya que cada evento tiene unas características determinadas en función de su tamaño, eventualidad y deporte o deportes practicados (40). Hasta donde alcanza nuestro conocimiento, no existen evidencias previas de evaluación de eventos deportivos propios de la especialidad de descenso de barrancos.

En relación al análisis de la repercusión del evento, los participantes reportaron que estos acontecimientos de pequeña escala tienen un impacto positivo para el territorio en el que se desarrolla. En comparación con otros estudios con residentes donde se diferencias diversos tipos de percepciones con respecto al evento (favorables, moderados y desfavorables) (20), incluso en los datos de los más favorables muestran puntuaciones más bajas que los participantes del presente estudio. También Parra-Camacho (43) encontraron percepciones positivas respecto a un evento deportivo por parte de los residentes, pero en niveles más bajos de los hallados en los participantes del evento evaluado en este estudio. El mismo autor, analizó también la percepción de los visitantes a un evento de pequeña escala encontrando también datos positivos (25).

Finalmente, sobre la estimación del gasto medio por participante, se obtuvo un importe superior a los 250 euros, el cual está en la tendencia de los eventos estudiados en España: 319,27 $€$ en el Campeonato de España Máster de Natación 2011 (44), 278,0 $€$ en el Campeonato de España Máster de Natación 2015 (19), en la Universiada de Granada 185,66 € (13), en el Medio Maratón de Oporto 88,55 $€$ participantes portugueses y 230,72 $€$ los participantes extranjeros, en el Maratón de 
Oporto $51,13 €$ los participante portugueses y $420,18 €$ los extranjeros (45). A pesar de no encontrar un gasto superior a otras modalidades deportivas, cabe destacar que el gasto por participante es bastante elevado y que posiblemente no es mayor por desarrollarse el evento en un entorno rural donde los precios de alojamientos y otros servicios son sensiblemente más económicos que en entornos urbanos.

Las principales conclusiones de este estudio son, en primer lugar, que la satisfacción de los participantes en el Campeonato de España de descenso de Cañones ha sido elevada, y que los participantes perciben al evento como un recurso que repercute de manera positiva en el territorio. La percepción y satisfacción de los participantes es un factor clave tanto para organizadores, que permitirá consolidar y mejorar el evento deportivo, como para la localidad que acoge el acontecimiento que se puede desarrollar como un destino turístico de referencia en la modalidad deportiva.

Asimismo, convenimos que la estimación de gasto de los participantes de los eventos deportivos es una herramienta útil del análisis del impacto económico, que permite a organizadores y administraciones argumentar las inversiones públicas y privadas necesarias para el desarrollo de estos acontecimientos.

\section{LIMITACIONES Y CAMINOS FUTUROS}

El presente estudio cuenta con la limitación de ser un evento puntual de una modalidad en la cual no se ha estudiado hasta ahora en términos de satisfacción y estimación de gasto de participantes. Además, al ser un evento de baja participación, la muestra es pequeña y puede limitar la obtención de resultados significativos. En relación a la estimación de gastos de los participantes, presenta limitaciones en relación al instrumento de medición por ítem y su método por rangos, que puede causar un sesgo en el cálculo total del participante.

En cuanto a líneas futuras de investigación, se considera necesario continuar esta línea de investigación mediante un análisis de próximos eventos organizados en la modalidad de descenso de cañones. Además, conviene completar el estudio con la satisfacción de público y sobre todo con la de los ciudadanos de la localidad que acoge el evento, para poder tener una visión más global de la repercusión positiva o negativa del evento en el territorio. Por otra parte, en relación al estudio del impacto económico de los eventos deportivos, es de interés, la realización de un estudio más profundo que añada los coeficientes multiplicadores y la interpretación apropiada del empleo, entre otras (46). Además, sería conveniente para próximos estudios analizar el gasto de los acompañantes así como recopilar los datos del organizador del evento 
en cuanto a ingresos obtenidos de patrocinadores o administraciones públicas externas al territorio y un desglose de los gastos realizados en la comarca y los realizados con proveedores externos, para poder realizar una estimación del impacto económico del evento en la zona.

\section{REFERENCIAS BIBLIOGRÁFICAS}

1. Ministerio de Cultura y Deporte. Anuario de estadísticas deportivas 2019. División de Estadística y Estudios, Secretaría General Técnica. 2019.

2. Kennedy H, Baker BJ, Jordan JS, Funk DC. Running recession: A trend analysis of running involvement and runner characteristics to understand declining participation. J Sport Manag. 2019; 33(3):215-28.

3. Theodorakis ND, Kaplanidou K, Karabaxoglou I. Effect of Event Service Quality and Satisfaction on Happiness Among Runners of a Recurring Sport Event. Leis Sci. 2015; 37(1):87-107.

4. Sánchez-Sáez JA. Los eventos deportivos como instrumento de desarrollo local. Cult Cienc y Deport. 2019; 14(41):91-2.

5. Cerro-Herrero D. Análisis de la calidad percibida y satisfacción de participantes en eventos deportivos. In: Catálogo de investigación joven en Extremadura. 2018; p. 66-70.

6. Richards G, Marques L, Mein K. Event design: Social perspectives and practices. Event Design: Social Perspectives and Practices. 2014.

7. Rodríguez C, Sánchez M. El Turismo de Eventos: Un análisis del perfil sociodemo- gráfico y comportamiento Del gasto turístico en función de la naturaleza del evento. Rev Port Estud Reg. 2018; 49:41-55.

8. Angosto S, López J, Díaz A. Una escala para la evaluación de la calidad pericibida por participantes en eventos deportivos populares (CAPPEP V2.0). J Sport Econ Manag. 2016; 6(2):69-84.

9. Shonk DJ, Chelladural P. Service quality, satisfaction, and intent to return in event sport tourism. J Sport Manag. 2008; 22(5):587-602.

10. Alguacil M, Pérez C, Aguado S, Boquera J. Does hosting a sport event promotes the city among athletes as a tourist destination? Sport TK-Revista Euroam Ciencias del Deport. 2016; 5(1):126-36.

11. Hyun M, Jordan JS. Athletic goal achievement: A critical antecedent of event satisfaction, re-participation intention, and future exercise intention in participant sport events. Sport Manag Rev. 2020; 23(2):256-70.

12. Bosch J, Murillo C, Raya J. a importancia económica del sector deportivo y el impacto económico de los eventos deportivos. In: E. Bandrés, J. F. Sanz, editors. Deporte y Economía. Funcas; 2019; p. 261-75. 
13. Roca A, González J, Porcel P, Cabello D. Impacto económico de los asistentes a la Universiada de Invierno del 2015 en la ciudad de Granada. Sport TK-Revista Euroam Ciencias del Deport. 2019; 8(1):7-12.

14. Pérez C. Análisis de la calidad del servicio en los eventos deportivos. calidad percibida y satisfacción de los espectadores y de los deportistas. Universidad de Valencia; 2010.

15. Angosto S, Ibáñez R, Morales V. Valoración de la calidad percibida, valor percibido y satisfacción de los participantes en una carrera popular de media distancia. V Congreso Iberoamericano de Economía del Deporte. El deporte: Dinamizador económico. 2014.

16. Angosto $S$. Diseño y validación de un cuestionario sobre calidad percibida en eventos deportivos populares (CAPPEP). Universidad de Murcia; 2014.

17. Prado V, Calabuig F. La medición de la calidad de servicio en eventos deportivos: modelos lineales vs. Qca. J Sport Econ Manag. 2016; 6(3):126-36.

18. Parra D, Elasri A, Triadó XM, Aparicio P. Análisis de la relación entre los beneficios y los costes percibidos y la satisfacción del residente con la celebración de un evento deportivo: Efecto mediador del valor percibido. Rev Psicol del Deport. 2016; 25(3):59-63.

19. Sánchez P, Salgado J, Rodríguez A, Barajas A. Impacto económico del XXI Campeonato de España "Open" de Invierno Master de Natación de Pontevedra 2015. Sport TK-Revista Euroam Ciencias del Deport. 2016; 5(Supl.):169-80.

20. Parra D, Calabuig F, Añó V, Ayora D, Núñez JM. El impacto de un evento deportivo mediano: percepción de los residentes de la comunidad de acogida. RETOS Nuevas Tendencias en Educ Física, Deport y Recreación. 2014; 26:88-93.

21. Calabuig F, Crespo J, Núñez J, Valantine I, Staskeviciute I. Role of perceived value and emotions in the satisfaction and future intentions of spectators in sporting events. Eng Econ. 2016; 27(2):221-9.

22. Zhou R, Kaplanidou K. Building social capital from sport event participation: An exploration of the social impacts of participatory sport events on the community. Sport Manag Rev. 2018; 21(5):491-503.

23. Balduck AL, Maes M, Buelens M. The social impact of the tour de france: Comparisons of residents' pre-and post-event perceptions. Eur Sport Manag Q. 2011; 2(2):91-113.

24. González RJ, Camacho D, Calabuig F, Añó V. Percepción de los residentes sobre el impacto del Mundobasket 2014 en Gran Canaria y apoyo a la celebración de eventos deportivos. Rev Iberoam Psicol del Ejerc y el Deport. 2016; 11(2):279-88.

25. Parra D, González RJ, Añó V, Ayora D. Percepción de los visitantes sobre el impacto social y sus intenciones con respecto a la celebración 
de un evento deportivo de pequeña escala. Rev Psicol del Deport. 2016; 25(1):93-6.

26. Gratton C, Dobson N, Shibli S. The economic importance of major sports events: A case-study of six events. Manag Leis. 2000; 5(1):17-28.

27. Preuss H. The Economic Impact of Visitors at Major Multi-sport Events. Eur Sport Manag Q. 2005; 5(3):281-301.

28. Porter PK, Fletcher D. The economic impact of the olympic games: Ex ante predictions and ex poste reality. J Sport Manag. 2008; 22(4):470-86.

29. Fernández-Alles MT. El Impacto Turístico de Los Eventos Deportivos: Un Estudio de Caso. Cuad Tur. 2014; 33:59-76.

30. Parra D, Calabuig F, Añó V, Ayora D, Núñez JM. El impacto de un evento deportivo mediano: percepción de los residentes de la comunidad de acogida. Retos Nuevas tendencias en Educ Física, Deport y Recreación. 2014; 26:88-93.

31. Medina FX, Abadía i Naudí S, Sánchez González R, Bantulà Janot J, Morejon Torné S. Deporte, cultura, turismo y medio ambiente: el caso de la trailwalker Intermón-Oxfam en Cataluña. Deport Cult Tur y medio Ambient el caso la trailwalker Intermón-Oxfam en Cataluña. 2015; 1(1):95-107.

32. Jiménez-Naranjo H V., Coca JL, Gutiérrez M, Sánchez MC. Cost-benefit analysis of sport events: The case of World Paddle Tour. Eur Res Manag Bus Econ. 2016; 22:131-8.

33. Jimenez-Naranjo H. Análisis del impacto socioeconómico de los eventos deportivos. Universidad de Extremadura; 2015.

34. Folgado-Fernández JA, Nunes P, López EL. Impacto del ocio activo y el deporte en el desarrollo del turismo. Un estudio comparativo de dos eventos con identidad territorial en Extremadura (España) y Setúbal (Portugal). VII Foro Internacional de Turismo Maspalomas Costa Canaria. 2019.

35. Agorreta J, Madruga M, Cerro D, Prieto-Prieto J. Impacto socioeconómico del turismo activo de eventos deportivos: Un estudio exploratorio para el caso del Valle del Jerte. ROTUR Rev Ocio y Tur. 2020; 14(2):88-104.

36. Henderson JC, Foo K, Lim H, Yip S. Sports events and tourism: The Singapore Formula One Grand Prix. Int J Event Festiv Manag. 2010; 1(1):60-73.

37. Sánchez P, Salgado J, Rodríguez A, Barajas A. Impacto económico del XXI Campeonato de España "Open" de Invierno Master de Natación de Pontevedra 2015. Sport TK-Revista Euroam Ciencias del Deport. 2016; 5(Supl.):169-80.

38. Veltri FR, Miller JJ, Harris A. Club Sport National Tournament: Economic Impact of a Small Event on a Mid-Size Community. Recreat Sport J. 2009; 33(2):119-28. 
39. Prieto-Prieto J, Madruga M, Cerro D. Los eventos deportivos. In: La situación del deporte en Extremadura. Una visión de AGEDEX. Asociación de Gestores del Deporte de Extremadura; 2017; p. 96-113.

40. Roca A, Cabello D, Gonzalez J, Courel J. Estudio de satisfacción de los asistentes a la Universiada de Invierno Granada 2015. Retos nuevas tendencias en Educ física, Deport y recreación. 2018; 33:247-51.

41. Murillo C, Carles M, Llop M, Moya X, Planas D. Campeonato Mundial de Patinaje Artístico Reus 2014: opinión de los participantes y legado económico. Sport Rev Euroam ciencias del Deport. 2016; 5(1):107-18.

42. Carreño-Clemente J. Impacto de la organización de eventos deportivos internacionales en Canarias sobre la actitividad turística. El Ironman de Lanzarote. Rev Atlántida Rev Canar Ciencias Soc. 2014; 6(2014-2015):16379.

43. Añó V, Calabuig F, Parra D. Impacto social de un gran evento deportivo: El Gran Premio de Europa de Fórmula 1. Cult Cienc y Deport. 2012; 7(19):53-65.

44. Méndez Rial B, Sánchez-Fernández P, Barajas Alonso Á. Impacto de eventos deportivos: el caso del Campeonato de España de natacion Pontevedra 2011. Rev Int derecho y gestión del Deport. 2012; 18:72-91.

45. Cortinhas R, Fernandes PO, Teixeira JP. Estimativa dos gastos diretos dos participantes envolvidos nas provas da meia maratona e da maratona do Porto 2015. XXVII Jornadas Espano-Lusas - Gestión Científica. Alicante; 2017.

46. Barajas A, Salgado J, Sánchez P. Problemática de los estudios de impacto económico de eventos deportivos. Estud Econ Apl. 2012; 30(2):441-61. 